\title{
Receiver Clock Jump and Cycle Slip Correction Algorithm for Single-Frequency GNSS
}

\section{Receivers}

\author{
John A. Momoh ${ }^{1}$, Santosh Bhattarai ${ }^{2}$ and Marek Ziebart ${ }^{3}$
}

\begin{abstract}
We introduce a simple single-band receiver clock jump and cycle slip (CJCS) detection and correction algorithm suitable for a standalone single-frequency Global Navigation Satellite System (GNSS) receiver. The real-time algorithm involves using an adaptive time differencing technique for the generation of adaptive differenced sequences of single-frequency code and phase observations. The sequences are used for determining thresholds and for the detection and determination of a receiver clock jump and cycle slips. The cycle slip values are fixed by rounding-up float values obtained via weighted least squares adjustment, following the elimination of the receiver's high-order clock drift at every epoch. The performance of this new technique was investigated with simulated cycle slip values and with different types of receiver clock jumps at millisecond and microsecond levels. It achieved $100 \%$ detection and correction of all types of receiver clock jumps; between 97 to $100 \%$ cycle slip detection; and between 96.9 to $100 \%$ cycle slip correction including cycle slips of \pm 1 cycle, for different rates of observations acquired by different fixed and mobile GNSS receivers. The algorithm thus facilitates precise timing and positioning on standalone low-cost single-frequency GNSS devices.
\end{abstract}

Keywords: single-frequency; receiver clock jump; cycle slip; adaptive time differencing.

\section{Introduction}

Global Navigation Satellite System (GNSS) receivers generate code and phase observations that can be affected by clock jumps and cycle slips (CJCS). If the CJCS problem is not effectively dealt with, it can result in reduced accuracy, prolonged convergence times and

\footnotetext{
${ }^{1}$ National Space Research and Development Agency (NASRDA), Nigeria; and Department of Electronic and Information Engineering, Landmark University, Oти-aran, Nigeria.

2 Space Geodesy and Navigation Laboratory, Dept. of Civil, Environmental and Geomatic Engineering, University College London, United Kingdom. Email: s.bhattarai@ucl.ac.uk

3 Space Geodesy and Navigation Laboratory, Dept. of Civil, Environmental and Geomatic Engineering, University College London, United Kingdom.
} 
unwanted frequent re-initializations, i.e., an overall reduced level of performance. Here, the authors present a new algorithm for dealing with this CJCS problem on a single-frequency GNSS receiver.

Several single-frequency cycle slip correction methods, which are either code-phase based, phase-only based or doppler-inclusive based, exist. A code-phase method is presented in Fath-Allah (2010). This method is limited by the usual code-level errors that often prevent the detection of small cycle slip values. Phase-only methods found in Jia and Wu (2001) and Cosser et al. (2004) are based on 3rd-order differencing or fitting, which have no means of detecting and eliminating receiver clock jumps or achieving reliable detection of cycle slips in data sets with low observation rates. The Doppler-inclusive methods, such as found in Ren et al. (2011), combine Doppler measurements with phase and/or code observations. One drawback with the Doppler-inclusive technique is the inability to detect receiver clock jumps, plus the fact that not all single-frequency GNSS chip sets provide Doppler measurements.

Known single-frequency techniques for addressing clock jumps are found in Momoh and Ziebart (2012), which involves phase differencing and code-based thresholding; and in Deo and El-Mowafy (2015), which is based on extrapolation and spline fitting of combined code and phase observations. These two methods are suitable for homogeneous clock jumps where receiver clock jumps are common to both the code and phase observations, but not for heterogeneous clock jumps where clock jumps manifest only on either the code or phase observation. The new CJCS algorithm presented uses single-frequency code and phase measurements from a standalone single-frequency GNSS receiver such as found in most lowcost GNSS chipsets.

\section{Phase and Code Observation Models}

As in Momoh and Ziebart (2012), we denote a GPS L1 receiver's raw code measurement as $P$; the accumulated carrier phase in cycles as $\Phi$; and the functional models for $P$ and the carrier phase observations in meters, $\psi$, as

$$
\begin{aligned}
P^{s} & =r^{s}+c \delta t^{r}-c \delta t^{s}+T^{s}+I^{s}+d^{s}+d^{r}+S_{o}^{s}+m_{P}^{s} \\
\psi^{s} & =\lambda \Phi^{s} \\
& =r^{s}+c \delta t^{r}-c \delta t^{s}+T^{s}-I^{s}+b^{r}+b^{s}+\lambda N^{s}+S_{o}^{s}+m_{\psi}^{s}
\end{aligned}
$$


As such, $\lambda$ is the wavelength given as $\lambda=c / f$, where $c=299792458 \mathrm{~m} / \mathrm{sec}$ is the speed of light; $r^{s}$ is the true geometric range between a receiver and satellite $s$, in meters; $\delta t^{s}$ and $\delta t^{r}$ are respectively the satellite and receiver clock offsets from the GPS system time in seconds; $T^{s}$ is the tropospheric delay in meters; $I^{s}$ is the $f$-dependent ionospheric delay in meters; and $S_{o}{ }^{s}$ is satellite $s$ orbital position error in the receiver-satellite direction, in meters. $N^{s}$ is the carrier phase integer ambiguity in cycles; $d^{r}, b^{r}$, and $d^{s}, b^{s}$ are the receiver and satellite hardware delays in meters; and $m_{\psi}^{s}$ and $m_{P}^{s}$ are the errors due to multipath and noise in the phase and code observations, respectively.

\section{Types of Receiver Clock Jumps}

Different types of receiver clock jumps, and at different jump magnitudes, exist for different receivers (Guo and Zhang 2014). The clock jump on observations can be classified into three types. Type 1 is indicated by a jump in the observed satellites' code observations only; Type 2 is indicated by a jump in the observed satellites' phase observations only, and Type 3 is indicated by jumps in both the code and phase observations of all observed satellites. The effect or manifestation of a clock jump on the observations is either heterogeneous, in the case of Types 1 and 2; or homogeneous, in the case of Type 3.

The clock jump can be an integer number of either a microsecond-level jump or a millisecond-level jump. The jump type and magnitude are usually unknown to users, manifesting differently in different receivers.

\section{Impacts of Receiver Clock Jump and Cycle Slip}

In line with Kim and Langley (2002), the contribution in a unit of distance, caused by a clock jump at epoch $t$, can be expressed as

$$
D\left(t+\tau_{r}\right)=\tau_{r} \rho^{s}+c \tau_{r}
$$

where $D\left(t+\tau_{r}\right)$ is the change in the observed code and/or phase observation in meters; $\tau_{r}$ is the value of the clock jump; $\rho^{s}$ is the range rate of the true geometric range between a receiver and $s$; and $c$ is the speed of light. Compared to the $c \tau_{r}$ term that is large and common across all observed satellites, the $\tau_{r} \rho^{s}$ term is negligibly small and different for each 
satellite. Thus, a clock jump contribution can be sufficiently represented and simulated by adding only the $c \tau_{r}$ term to (1) and (2).

On the occurrence of a cycle slip at $t$, the integer ambiguity changes from $N^{s}(t-1)$ to $N^{s}(t)=N^{s}(t-1)+\Delta N^{s}$, where $\Delta N^{s}$ is the cycle slip magnitude that could be an integer value from 1 to millions of cycles (de Lacey et al. 2011). Thus, considering clock jumps and cycle slips, the modified functional models for $P$ and $\psi$ become:

$$
\begin{aligned}
& P^{s}(t)=r^{s}+c\left(\delta t^{r}+\tau_{r}\right)-c \delta t^{s}+T^{s}+I^{s}+d^{s}+d^{r}+S_{o}^{s}+m_{P}^{s} \\
& \psi^{s}=\lambda \Phi^{s} \\
& \quad=r^{s}+c\left(\delta t^{r}+\tau_{r}\right)-c \delta t^{s}+T^{s}-I^{s}+b^{r}+b^{s}+\lambda\left(N^{s}+\square N\right)+S_{o}^{s}+m_{\psi}^{s}
\end{aligned}
$$

A homogenous clock jump would not degrade a positioning solution, but it would be necessary, in real time, to detect and correct homogenous clock jumps for receiver clock modeling and prediction (Weinbach and Schon 2011; Deo and El-Mowafy 2015). Heterogeneous clock jumps can degrade positioning significantly.

\section{Adaptive Time Differencing}

A sequence, $X=\left[x_{1}, x_{2}, \ldots, x_{n}\right]$, which contains phase or code observations from consecutive epochs, will sometimes include observations that are affected by clock jumps and/or cycle slips. The Adaptive Time Differencing (ATD) technique introduced in Momoh (2013) aids the detection of clock jumps and cycle slips in such sequences. To observe how the ATD technique works, consider Table 1, where we show mean values and standard deviations for 30-epoch-length sequences of raw phase observables and their differenced sequences (with increasing order of differencing, $d$ ), obtained for different GPS satellites observed with different receivers.

Table 1 Statistics of phase sequences and their ADSs obtained from receivers with different observation rates.

\begin{tabular}{|c|c|c|c|}
\hline MBAR, PRN28 & u-blox, PRN9 & Novatel, PRN14 & diff \\
\hline
\end{tabular}




\begin{tabular}{|c|c|c|c|c|c|c|}
\hline \multicolumn{2}{|c|}{$\tau=30$ seconds } & \multicolumn{2}{c|}{$\tau=1$ second } & \multicolumn{2}{c|}{$\tau=\mathbf{0 . 1}$ second } & order \\
\hline $\boldsymbol{\sigma}$ & $\boldsymbol{\mu}$ & $\boldsymbol{\sigma}$ & $\boldsymbol{\mu}$ & $\boldsymbol{\sigma}$ & $\boldsymbol{\mu}$ & $\boldsymbol{d}$ \\
$\left(\mathrm{m} / \tau^{\mathrm{d}}\right)$ & $\left(\mathrm{m} / \tau^{\mathrm{d}}\right)$ & $\left(\mathrm{m} / \tau^{\mathrm{d}}\right)$ & $\left(\mathrm{m} / \tau^{\mathrm{d}}\right)$ & $\left(\mathrm{m} / \tau^{\mathrm{d}}\right)$ & $\left(\mathrm{m} / \tau^{\mathrm{d}}\right)$ & \\
\hline $1.20 \mathrm{E} 5$ & $2.14 \mathrm{E} 7$ & $1.13 \mathrm{E} 3$ & $2.0 \mathrm{E} 7$ & $3.22 \mathrm{E} 2$ & $2.2 \mathrm{E} 7$ & 0 \\
\hline $6.30 \mathrm{E} 2$ & $1.41 \mathrm{E} 4$ & $9.21 \mathrm{E}-1$ & $1.3 \mathrm{E} 2$ & $2.69 \mathrm{E}-3$ & $3.7 \mathrm{E} 1$ & 1 \\
\hline $2.53 \mathrm{E} 0$ & $7.69 \mathrm{E} 1$ & $3.61 \mathrm{E}-2$ & $1.1 \mathrm{E}-1$ & $\mathbf{3 . 8 5 E}-3$ & $-1.4 \mathrm{E}-5$ & $\mathbf{2}$ \\
\hline $1.74 \mathrm{E}-1$ & $-3.27 \mathrm{E}-1$ & $\mathbf{5 . 3 6 E}-2$ & $2.5 \mathrm{E}-3$ & $6.64 \mathrm{E}-3$ & $2.6 \mathrm{E}-4$ & $\mathbf{3}$ \\
\hline $\mathbf{2 . 8 0 E}-1$ & $-9.70 \mathrm{E}-3$ & $9.32 \mathrm{E}-2$ & $-1.2 \mathrm{E}-3$ & $11.6 \mathrm{E}-3$ & $9.5 \mathrm{E}-4$ & $\mathbf{4}$ \\
\hline $4.87 \mathrm{E}-1$ & $2.38 \mathrm{E}-4$ & $17.3 \mathrm{E}-2$ & $-4.7 \mathrm{E}-9$ & $21.3 \mathrm{E}-3$ & $2.5 \mathrm{E}-4$ & 5 \\
\hline
\end{tabular}

The symbol $\tau$ denotes the observation epoch interval in seconds and the statistical computations exclude the last index (i.e., the last epoch) values of the sequences. Table 1 shows the decreasing 1-sigma trend prior to the transition-sigma values (in bold), and the increasing 1-sigma trend thereafter. The three differencing orders: 4, 3 and 2 (indicated in bold), are the respective optimum orders of differencing for the 1/30,1 and $10 \mathrm{~Hz}$ data sets since they are the orders corresponding to the transition-sigma values. The corresponding noise-like differenced sequence at an optimum $d$ is here referred to as an Adaptive Differenced Sequence (ADS).

Following from Momoh (2013), the phase ADS value is given as:

$$
\Delta^{d_{s}} \overline{\psi^{s}} \square c \square^{d_{s}} \delta t^{r}+\lambda \square N^{s}+\square^{d_{s}} m_{\psi}^{s}
$$

while the code ADS value is given as:

$$
\Delta^{d_{s}} P^{s} \square c \square^{d_{s}} \delta t^{r}+\square \square^{d_{s}} m_{P}^{s}
$$

where $\Delta N^{s}$ and $\Delta d_{s} \delta t^{r}$ denote a cycle slip and the combined receiver high-order clock drift and jump, respectively, $\Delta d_{s} m_{P}^{s}$ and $\Delta d_{s} m_{\psi}^{s}$ are the levels of the differenced code and phase noise, and $\Delta d_{s} m_{P}^{s} \gg \Delta d_{s} m_{\psi}^{s}$. 
Fig. 1 shows the phase ADSs obtained from the first 30-epoch-length $1 \mathrm{~Hz}$ raw phase observations from PRNs 28, 8, 11, 13, 4 and 17, simultaneously observed at static station MBAR, on day 170 of 2009 . The same $d_{s}=3$ resulted for all six satellites. To depict slip signatures of small cycle slip values on an ADS, cycle slips magnitudes of $+1,+2$ and -1 cycles were simulated at the last epoch for PRNs 28, 8 and 11 respectively. We presume the exhibited correlation is mainly due to the common high-order receiver clock drift, $\varepsilon_{c k}=c \Delta^{d} \delta t^{r}$

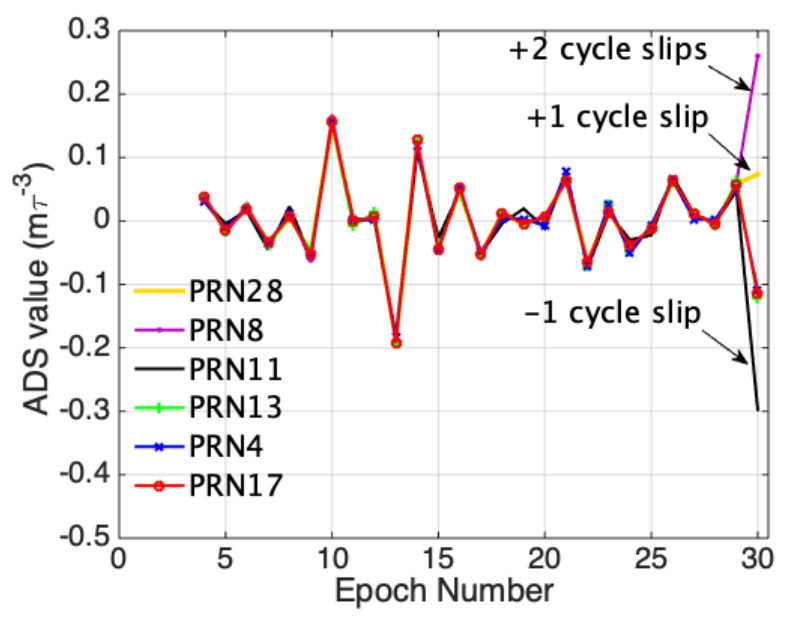

Fig. 1 Plots of phase ADSs obtained from simultaneously observed PRNs by a single receiver. The standard deviations of the ADSs, in units of $\mathrm{m} / \tau^{3}$, are $0.064,0.067,0.065$, 0.066, 0.065 and 0.066 for PRNs $28,8,11,13,4$ and 17 , respectively.

Fig. 2 shows the time series of estimated high-order receiver clock drift values for a ublox receiver driven by a crystal oscillator; and for the AMC2 reference station receiver (at Colorado, USA) that is driven by an external hydrogen maser, which is an atomic frequency standard that ensures high frequency stability. The range of values of $\varepsilon_{c k}$ for the quartz clock $\left(\sigma_{c l k}=2.82 \mathrm{~cm} / \mathrm{s}^{3}, d_{s}=3\right)$ is larger than that for the atomic clock $\left(\sigma_{c l k}=1.9 \mathrm{~mm} / \mathrm{s}^{3}, d_{s}=3\right)$. For lower rate data sets (i.e. where $\tau \geq 30 \mathrm{~s}$ ) the optimal differencing order is higher, i.e. $d_{s} \geq 3$, and the resulting phase ADSs usually become uncorrelated and unsuitable for estimating $\varepsilon_{c k}$ or for detecting small-valued cycle slips. 

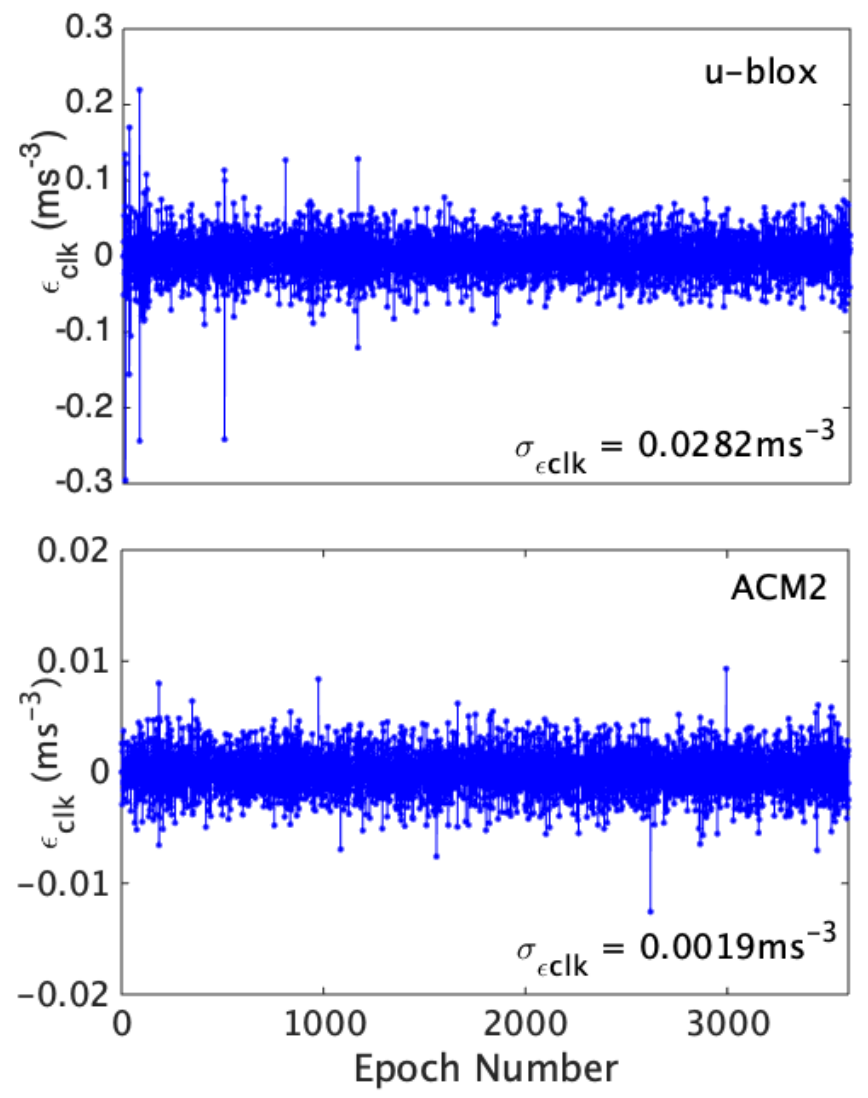

Fig. 2 Plots of the estimated high-order receiver clock drifts with $\tau=1$ second for $\mathrm{u}$-blox (top) and AMC2 (bottom)

\section{Receiver Clock Jump and Cycle Slip Detection}

The detection procedure of the CJCS algorithm is presented in the following steps:

(a) For each of the $N$ observed satellites at $t$, obtain the code and phase M-length sequences, each sequence being a time series of CJCS-free observations from the last M-1 epochs, and the current epoch's observation. For reliable statistical inference, M should be between 15 and 30 consecutive epochs.

(b) Perform ATD on all phase and code sequences. Determine the optimum order of differencing for each sequence and generate the phase and code ADSs. The optimum order is the arithmetic mode of orders of differencing of the phase sequences. 
(c) From each ADS, extract the last value. Use the extracted values to populate two row vectors, $V_{\psi}$ and, $V_{P}$, both of length $N$, which contain extracted values from the phase and code ADSs, respectively. Excluding the last values, obtain the 1-sigma $\sigma_{\psi}^{s}$ and $\sigma_{p}^{s}$ values for each phase and code ADS, respectively. Set an epoch-specific threshold value, $T_{d}$, for phase cycle slip detection as

$$
T_{d}=d_{s}\left(\left|\mu_{\varepsilon c l k}\right|+\sigma_{\varepsilon c l k}\right)
$$

where the almost zero-value $\mu_{\varepsilon c k}$, and $\sigma_{\varepsilon c k}$, are the mean and standard deviation of the corresponding $M$-length time series of $\varepsilon_{c l k}$.

(d) With $V_{\psi}$, generate the relative-difference matrix, $R_{D}$, whose $(m, n)$ index contains the absolute value of the difference between the $m^{\text {th }}$ and $n^{\text {th }}$ values of $V_{\psi}$, for each $m=1,2, . ., N$ and $n=1,2, . ., N$.. As an example, suppose we use the ADSs for PRNs 28, 8, 11, 13, 4 and 17, as shown in Fig. 1. In this case, $V_{\psi}$ and $R_{D}$ are:

$$
\begin{gathered}
V_{\psi}=\left[\begin{array}{c}
0.074 \\
0.260 \\
-0.297 \\
-0.121 \\
-0.110 \\
-0.115
\end{array}\right] \\
R_{D}=\left[\begin{array}{cccccc}
0 & 0.186 & 0.371 & 0.195 & 0.184 & 0.189 \\
& 0 & 0.557 & 0.381 & 0.371 & 0.375 \\
& & 0 & 0.176 & 0.187 & 0.182 \\
& & & 0 & 0.011 & 0.006 \\
& & & & 0 & 0.005 \\
& & & & 0
\end{array}\right]
\end{gathered}
$$

where the values in rows $1,2,3,4,5$ and 6 of $V_{\psi}$ relate to the PRNs 28, 8, 11, 13, 4 and 17 respectively.

(e) If the maximum value in $R_{D}$ is greater than or equal to $0.5 \lambda$, i.e. $R_{D}^{\max } \geq 0.5 \lambda$, we suspect that at least one cycle slip satellite exists, otherwise we assume there are none. If a cycle slip is suspected, we find all entries where $R_{D}(m, n) \geq 0.5 \lambda$ (i.e. $\geq 0.0951$ in our example), then add a +1 count to both satellite $m$ and $n$, and use the final count to populate a slip count vector, $\mathrm{S}$. In our example, $S=[5,5,5,3,3,3]$ indicating a count of 5 for PRNs 28,8 and 11 , and a count of 3 for PRNs 13, 4 and 17. 
(f) When cycle slip values among the slipped satellites are unique, the maximum entry value in $S$ is $N-1$, and each of the entries is either the maximum or the minimum value. The minimum value indicates the number of slipped satellites, which are the satellites with the maximum-value entries. Thus, $S=[5,5,5,3,3,3]$ indicates the 3 cycle-slipped satellites PRN 28,8 , and 11 .

(g) Other combinations of entry values, such as $S=[5,4,4,3,2,3, . ., 4]$ occur when the cycle slip values are not unique, i.e. two or more satellites have the same cycle slip value. In such cases, the values in $S$ would range from a minimum value, $\alpha$, to a maximum value, $\Omega=N-1$, and the slipped satellites are detected in two steps. First, we identify $\alpha$, and collate the satellites that correspond to the $\alpha$ maximum values in $S$ as slipped satellites. As an example, if $S=[5,4,4,3,2,3]$ for $N=6$, then $\alpha=2$ and $\Omega=5$, then the three satellites corresponding to the 5, 4, 4 entry values are selected as slipped satellites. The second step involves comparing the absolute value of each of the values in $V_{\psi}$ with the current epoch's computed threshold $T_{d}$. A cycle slip is suspected on the $k^{\text {th }}$ satellite if $\left|V_{\psi}(k)\right| \geq T_{d}$, for $k=1,2, . ., N$. This step provides some more slipped satellites. Thus, the suspected slipped satellites are the combined satellites obtained from these two steps.

(h) When the $S$ vector contains only maximum value entries, such as $S=[\Omega, \Omega, \Omega, . ., \Omega]$, it indicates that all satellites, except any that may have $\left|V_{\psi}(k)\right|<T_{d}$ for $k=1,2, . ., N$, have cycle slips. When the vector has at least one zero value entry, it is presumed that there are no cycle slip satellites.

(i) Having identified the cycle-slipped satellites, the remaining $K$ non-slipped satellites' observables in $V_{\psi}$ are then used for testing for a phase clock jump. When no satellite indicates cycle slip or for the rare situation when all satellites indicate cycle slips $K=N$ and all $N$ values in $V_{\psi}$ are used in the phase clock jump detection. For code observations, all $N$ values in $V_{P}$ are used for code clock jump detection.

(j) Fixed thresholds are set for clock jump detection and validation. A clock jump detection threshold, $D_{t h}=0.75 \mathrm{c} \times 10^{-6} \mathrm{~m}$; and a tolerance threshold, $T_{t h}=0.25 \mu \mathrm{s}$ for microsecond-level and $T_{t h}=0.25 \times 10^{-3} \mathrm{~ms}$ for millisecond-level jumps, respectively, are used. Thus, a clock jump is detected on the phase and code using 


$$
\operatorname{Jump}_{\psi}(k)=\left\{\begin{array}{ll}
1, & \text { if }\left|V_{\psi}(k)\right| \geq D_{t h} \\
0, & \text { otherwise }
\end{array}, k=1,2, . ., K\right.
$$

and

$$
\operatorname{Jump}_{P}(k)=\left\{\begin{array}{ll}
1, & \text { if }\left|V_{P}(k)\right| \geq D_{t h} \\
0, & \text { otherwise }
\end{array}, k=1,2, . ., N\right.
$$

respectively, with $*$ denoting the subscript $\psi$ or $P$. $\operatorname{Jump}_{*}(k)=1$ indicate detection of clock jump on the $k^{\text {th }}$ satellite. For every $\operatorname{Jump}_{*}(k)=1$, we obtain the corresponding $V_{*}(k)$ observable as $J_{*}^{d}(k)=V_{*}(k)$. Thus, we can have detected clock jump observable vectors, $J_{*}^{d}(k)$

\section{Validation and Determination of Clock Jumps}

The validation and determination of a clock jump value follow thus:

(i) First, we obtain the integer value $z_{*}(k)=\operatorname{round}\left\{J_{*}(k) /\left(c \times 10^{-6}\right)\right\}$, signifying the roundingup of $\{$.$\} to an integer. If z_{*}(k) \geq 999$, this indicates a millisecond-level jump. In that case, $z_{*}(k)$ is recomputed as $z_{*}(k)=\operatorname{round}\left\{J_{*}(k) /\left(c \times 10^{-3}\right)\right\}$, for all observables in $J_{*}^{d}(k)$.

(ii) Each of the detected clock jump observables in $J_{*}^{d}(k)$ are then validated by checking the remainder value, $R_{*}(k)=J_{*}^{d}(k)-z_{*}(k)$, against the microsecond or millisecond-level $T_{t h}$ value. A validated phase or code clock jump is obtained as

$$
J_{\psi}^{v}(k)=z_{\psi}(k), \quad \text { if } \quad\left|R_{\psi}(k)\right|<T_{t h}
$$

Thus $J_{*}^{v}$ is a vector of validated integer numbers of clock jump value(s).

(iii) If the number of non-zero values in $J_{*}^{v}$ is at least half the number of satellites used in the clock jump detection, a clock jump is confirmed and its value, $J_{*}$ is computed as

$$
J_{*}=\operatorname{mode}\left(J_{*}^{v}\right)
$$

where mode is the arithmetic mode operator. Outliers, if any, are excluded from the values in $J_{*}^{v}$ before the mode value is computed. There is no clock jump if $J_{*}^{v}$ is an empty vector or it 
contains zero values more than half of the number of satellites used in the clock jump detection.

\section{Cycle Slip Determination}

The clock-jump-free vector, $\bar{V}_{\psi}=V_{\psi}-\beta c J_{\psi /}(t)$, where $\beta=10^{-6}$ or $\beta=10^{-3}$ for microsecond or millisecond jump respectively, is obtained. If $\mathrm{Q}$ is the number of cycle-slipped satellites detected at $t$, the value of $\varepsilon_{c k}(t)$ is estimated in the three possible cases:

(i) When $Q=0, \varepsilon_{c l k}(t)$ is estimated as the weighted average of the values in $\overline{V_{\psi}}$ as

$$
\varepsilon_{c l k}(t)=\frac{\sum_{k=1}^{N} w_{k} \bar{V}_{\psi}(k)}{\sum_{k=1}^{N} w_{k}}
$$

where $w_{k}=\left(1 / \sigma_{k}\right)^{2}$ and $\sigma_{k}$ being the standard deviation of the ADS for the $k^{\text {th }}$ satellite.

(ii) When $1 \leq Q<N, \varepsilon_{c k}(t)$ is estimated from the N-Q non-slipped satellites' values in $\overline{V_{\psi}}$ as

$$
\varepsilon_{c l k}(t)=\frac{\sum_{k=1}^{N-Q} w_{k} \bar{V}_{\psi}(k)}{\sum_{k=1}^{N-Q} w_{k}}
$$

(iii) When $Q=N$, we compute the mean of the immediate past $M$-length time series of $\varepsilon_{c k}$ as the estimated value for $\varepsilon_{c l k}(t)$ :

$$
\varepsilon_{c l k}(t)=\frac{1}{M} \sum_{\tau_{0}=t-M}^{t-1} \varepsilon_{c l k}\left(\tau_{0}\right)
$$

The cycle slip values of the detected $q=1,2, . ., Q$ cycle-slipped satellites are subsequently determined. First, from among the slipped satellites, the highest-elevation satellite is identified as the reference satellite, say the satellite corresponding to $q=1$ with a cycle slip value of $\Delta N_{1}$. Then, new sequences, $U_{1, q}$, for $q=2,3, . ., Q$ are formed, where each sequence contains the values of the corresponding index-to-index difference between the ADS of reference satellite $(q=1)$ and the ADS of the other cycle slip satellites.

Defining 


$$
\begin{gathered}
H=\left[\begin{array}{cccccc}
\lambda & -\lambda & 0 & & 0 & 0 \\
\lambda & 0 & -\lambda & \ldots & 0 & 0 \\
& & \vdots & & & \\
\lambda & 0 & 0 & & -\lambda & 0 \\
\lambda & 0 & 0 & & 0 & -\lambda \\
\lambda & 0 & 0 & & 0 & 0 \\
0 & \lambda & 0 & & 0 & 0 \\
0 & 0 & \lambda & 0 & 0 \\
0 & 0 & 0 & & \lambda & 0 \\
0 & 0 & 0 & & 0 & \lambda
\end{array}\right] \\
\Delta X=\left[\begin{array}{c}
\vdots \\
\Delta N_{1} \\
\Delta N_{2} \\
\Delta N_{3} \\
\vdots \\
\Delta N_{Q-1} \\
\Delta N_{Q}
\end{array}\right] ; \quad \text { and } \\
\end{gathered}
$$

the relationship

$$
H \Delta X=Y
$$

holds, where $\bar{U}_{1, q}$ is the last value of $U_{1, q}$ that has a standard deviation $\sigma_{1, q}$; $\dot{V}_{\psi}(q)=\bar{V}_{\psi}(q)-\varepsilon_{c l k}(t) ; \mathrm{H}$ is a $(2 Q-1) \times Q$ matrix; $\Delta X$ is a $Q \times 1$ vector of the unknown cycle slips; and $Y$ is a $2 Q-1$ vector of observables without $\varepsilon_{c k}(t)$. With a diagonal weight matrix $W=\operatorname{diag}\left[\frac{1}{\sigma_{1,2}^{2}}, \frac{1}{\sigma_{1,3}^{2}}, \ldots, \frac{1}{\sigma_{1, Q-1}^{2}}, \frac{1}{\sigma_{1, Q}^{2}}, \frac{1}{\sigma_{1}^{2}}, \frac{1}{\sigma_{2}^{2}}, \frac{1}{\sigma_{3}^{2}}, \ldots, \frac{1}{\sigma_{Q-1}^{2}}, \frac{1}{\sigma_{Q}^{2}}\right]$

the vector $\Delta X=\left[\Delta N_{1}, \Delta N_{2}, \Delta N_{3}, \ldots, \Delta N_{Q-1}, \Delta N_{Q}\right]^{T}$ of estimated float values of the unknown cycle slips is obtained by the method of weighted least squares (Markovsky and Huffel 2007).

The integer vector, $\Delta \bar{X}=\left[\Delta \bar{N}_{1}, \Delta \bar{N}_{2}, \Delta \bar{N}_{3}, . ., \Delta \bar{N}_{Q-1}, \Delta \bar{N}_{Q}\right]^{T}$, is obtained by rounding-up the float values in $\Delta X$ to the nearest integer values, thus obtaining the cycle slip fixed integer values for the slipped satellites as $\Delta \bar{N}_{1}, \Delta \bar{N}_{2}, \ldots, \Delta \bar{N}_{Q}$. 


\section{Receiver Clock Jump and Cycle Slip Correction}

For Type 1 clock jumps detected in past epoch(s), the phase observations of all satellites at the current epoch are updated with the accumulated code clock jump (since the $M^{\text {th }}$ epoch), to give $\overline{\psi^{s}}(t)$ for each $s$ as

$$
\overline{\psi^{s}}(t)=\psi^{s}(t)+c \sum_{\tau_{0}=M}^{t-1} J_{P}\left(\tau_{0}\right)
$$

For Type 2 clock jumps detected in past epoch(s), the code observations of all satellites at the current epoch are updated with the accumulated phase clock jump (since the $M^{\text {th }}$ epoch), to give $\overline{P^{s}}(t)$ for each $s$ as

$$
\overline{P^{s}}(t)=P^{s}(t)+c \sum_{\tau_{0}=M}^{t-1} J_{\psi}\left(\tau_{0}\right)
$$

A post-detection clock jump correction is subsequently performed at $t$ : for Type 1 , the phase observation is corrected for the $J_{P}(t)$ code clock jump as

$$
\overline{\overline{\psi^{s}}}(t)=\overline{\psi^{s}}(t)+c J_{P}(t)
$$

while the time-series of the previous CJCS-corrected phase observables, $\hat{\psi}(1: t-1)$, is corrected with the same $J_{P}(t)$ as

$$
\overline{\overline{\psi^{s}}}(1: t-1)=\psi^{s}(1: t-1)+c . J_{P}(t)
$$

for each satellite. For Type 2, the correction on the code is obtained as:

$$
\begin{aligned}
\overline{\overline{P^{s}}}(t) & =\overline{P^{s}}(t)+c . J_{\psi}(t) \\
\overline{\overline{P^{s}}}(1: t-1) & =\overline{\overline{P^{s}}}(1: t-1)+c J_{\psi}(t)
\end{aligned}
$$

For Type 3, $J_{\psi}(t)=J_{P}(t)$ and there is no correction required. However, if receiver clock modeling is required, updates of the past observations will be necessary.

The cycle slip on a $q^{\text {th }}$ satellite $(q=1,2, . ., Q)$, with cycle slip value $\Delta \bar{N}_{q}$, is corrected by updating the past time series of the clock-jump-corrected phase observable, $\overline{\psi^{q}}(1: t-1)$, to give 


$$
\psi^{q}(1: t-1)=\overline{\overline{\psi^{q}}}(1: t-1)+\lambda \Delta \bar{N}_{q}
$$

as the CJCS-corrected time series phase observable.

\section{Tests, Results and Discussion}

The proposed algorithm was tested under different conditions. The cycle slip and clock jump detection and correction capability was investigated by way of simulation, using actual single-frequency observations. For the cycle slip tests, known but randomly generated integer cycle slip values were applied to the phase observations of a randomly selected number of observed satellites at every five epochs observation interval, starting from the first $M=30$ epochs. The responsiveness and accuracy of the ATD algorithm to cycle slip detection and determination was investigated by using randomly generated non-zero integer cycle slip values between -100 and +100 , which include small cycle slip values that are usually more challenging to accurately detect and fix. Using different single-frequency phase observations at different rates and by different fixed and mobile GNSS receivers set at elevation mask angle of $5^{0}$, the results shown in Table 2 were obtained.

Table 2 Simulated single-frequency cycle slip results using observations from different receivers operating at different observation rates

\begin{tabular}{c|c|c|c|c|c|c}
\hline Receiver & $\begin{array}{c}\text { Clock } \\
\text { Type }\end{array}$ & $\begin{array}{c}\text { Data } \\
\text { Rate }\end{array}$ & $\begin{array}{c}\text { Tested } \\
\text { No. of } \\
\text { cycle } \\
\text { slips }\end{array}$ & $\begin{array}{c}\text { Quantity } \\
\text { of cycle } \\
\text { slips } \\
\text { Detected }\end{array}$ & $\begin{array}{c}\text { Quantity } \\
\text { of cycle } \\
\text { slips fixed }\end{array}$ & $\begin{array}{l}\text { Maximum. } \\
\text { fix error } \\
\text { (cycles) }\end{array}$ \\
\hline u-blox & quartz & 1 & 4480 & 4480 & 4469 & +1 \\
(fixed) & & 1 & 4255 & 4255 & 4255 & \\
\hline AMC2 & atomic & $100 \%)$ & $(99.8 \%)$ & \\
(fixed) & standard & & & $(100 \%)$ & $(100 \%)$ & \\
\hline
\end{tabular}




\begin{tabular}{c|c|c|c|c|c|c}
\hline $\begin{array}{c}\text { Novatel } \\
\text { (fixed) }\end{array}$ & quartz & 10 & 4471 & 4471 & 4471 & 0 \\
\hline MBAR & quartz & $1 / 30$ & 4325 & 4201 & 4150 & \pm 2 \\
(fixed) & & & & $(100 \%)$ & $(100 \%)$ & \\
\hline SHIP & quartz & 1 & 2781 & 2780 & 2695 & \pm 2 \\
(mobile) & & & & $(99.7 \%)$ & $(96.9 \%)$ & \\
\hline
\end{tabular}

The u-blox (EVK-6 evaluation kit) is a standard single-frequency receiver driven by a quartz crystal oscillator. AMC2, Novatel, MBAR and SHIP have different dual-frequency receivers, but only their L1 single-frequency observations are used. For AMC2 and MBAR, both IGS stations, we use data from day 170 of 2009. Novatel was fixed and its data was obtained on day 90 of 2017 in Abuja, Nigeria. SHIP was mobile and its data was obtained on day 122 of 2008 in Harwick Harbor, United Kingdom, on a moving ship. As indicated in Table 2, the AMC2 receiver in Colorado is driven by an external hydrogen maser while the rest are driven by quartz crystal oscillators. From each receiver, 3600 epochs of observations were processed in real-time kinematic mode. A complete $100 \%$ detection and correction of all the cycle slips simulated for AMC2 and the Novatel was achieved. There were 107 and 112 different epochs when all observed satellites were affected with cycle slips for AMC2 and Novatel respectively. For the other receivers, between 97 to $100 \%$ of detection and between 96 to $99.8 \%$ of correct fixing were achieved, with a maximum fixed error of \pm 2 cycles. For the u-blox receiver, all observed satellites were affected with cycle slips at 105 different epochs. From this, one epoch of $\mathrm{N}=11$ satellites were wrongly fixed by a maximum error of +1 cycle each, resulting in the $99.8 \%$ correct fix. The ATD technique achieved $97 \%$ and $99.7 \%$ detection for MBAR and SHIP, respectively. The remaining 3\% undetected slips in MBAR's 30 second-interval data comprised mainly cycle slip values of one or two cycles.

The ATD technique did not detect 1 cycle slip with a value of -1 cycle, out of 2781 cycle slips simulated for SHIP, possibly due to a destructive combination of the -1 cycle slip value with the dynamics of the moving ship. The fixing accuracy for MBAR was possibly affected by the relatively high value of the ADSs, resulting in estimated high-value (up to meter level) 
receiver clock drifts. Such large ADS values from low rate observations usually result from variations in true geometric range, the ionosphere and receiver clock drift in consecutive epochs, preventing small cycle slip detection, especially during geomagnetic storms or around the geomagnetic equator. For MBAR and SHIP, most of the incorrect fixes occurred in the 110 and 76 distinct epochs, respectively, when all satellites observed had cycle slips. For both cases, the fixing errors were less than \pm 2 cycles. The results indicate that it is easier to achieve $100 \%$ detection and correction of cycle slips with the ATD technique when the high-order receiver clock drift is very small (millimeter level) such as obtained for the AMC2 receiver driven by an atomic frequency standard (see bottom plot of Fig. 2), or when observations are obtained at higher rates (see Table 1) as with the Novatel receiver that generated observations at $10 \mathrm{~Hz}$.

We also investigated the performance of the algorithm in dealing with the three types of receiver clock jumps by simulating both millisecond and microsecond-level clock jumps. The equivalent magnitudes of randomly selected clock jump values, in a unit of distance, were added to the phase and/or code observations of all satellites, depending on the type of clock jump simulated. The simulation was done at every 100-epoch interval, beginning from the $30^{\text {th }}$ epoch of observations from the u-blox single-frequency receiver. In these tests, for which the result are shown in Table 3, cycle slip simulation was not done so as to assess the performance of the algorithm in dealing with clock jumps only. The accumulated clock jump is the sum of the 36 randomly selected clock jump integer values, in the simulation based on 3600 epochs of observation from the u-blox receiver. Fig. 3 shows the clock jump value at every clock jump epoch and the accumulated clock jump at such epochs.

Table 3 Results of simulated millisecond- and microsecond-level clock jumps on the u-blox single-frequency receiver.

\begin{tabular}{|c|c|c|c|c|c|c|c|}
\hline \multicolumn{4}{|c|}{ Millisecond-level clock jump } & \multicolumn{4}{|c|}{ Microsecond-level clock jump } \\
\hline $\begin{array}{c}\text { Clock } \\
\text { jump } \\
\text { type }\end{array}$ & $\underset{\text { jump }}{\sum}$ & $\begin{array}{c}\text { Jump } \\
\text { detection } \\
\text { and } \\
\text { correction }\end{array}$ & $\begin{array}{l}\text { Clock } \\
\text { jump } \\
\text { range }\end{array}$ & $\begin{array}{c}\text { Clock } \\
\text { jump } \\
\text { type }\end{array}$ & $\underset{\text { jump }}{\sum}$ & $\begin{array}{c}\text { Jump } \\
\text { detection } \\
\text { and } \\
\text { correction }\end{array}$ & $\begin{array}{l}\text { Clock } \\
\text { jump } \\
\text { range }\end{array}$ \\
\hline Type 1 & $26 \mathrm{~ms}$ & Det: $100 \%$ & -10 to & Type 1 & $45 \mu \mathrm{s}$ & Det: $100 \%$ & -10 to \\
\hline
\end{tabular}




\begin{tabular}{|c|c|c|c|c|c|c|c|}
\hline & & $\begin{array}{l}\text { Cor: } 100 \% \\
\text { False }=0\end{array}$ & $10 \mathrm{~ms}$ & & & $\begin{array}{c}\text { Cor: } 100 \% \\
\text { False }=0\end{array}$ & $10 \mu \mathrm{s}$ \\
\hline Type 2 & $47 \mathrm{~ms}$ & $\begin{array}{l}\text { Det: } 100 \% \\
\text { Cor: } 100 \% \\
\text { False }=0\end{array}$ & $\begin{array}{l}-10 \text { to } \\
10 \mathrm{~ms}\end{array}$ & Type 2 & $48 \mu \mathrm{s}$ & $\begin{array}{c}\text { Det: } 100 \% \\
\text { Cor: } 100 \% \\
\text { False }=0\end{array}$ & $\begin{array}{l}-10 \text { to } \\
10 \mu \mathrm{s}\end{array}$ \\
\hline Type 3 & $72 \mathrm{~ms}$ & $\begin{array}{l}\text { Det: } 100 \% \\
\text { Cor: } 100 \% \\
\text { False }=0\end{array}$ & $\begin{array}{l}-10 \text { to } \\
10 \mathrm{~ms}\end{array}$ & Type 3 & $64 \mu \mathrm{s}$ & $\begin{array}{c}\text { Det: } 100 \% \\
\text { Cor: } 100 \% \\
\text { False }=0\end{array}$ & $\begin{array}{l}-10 \text { to } \\
10 \mu \mathrm{s}\end{array}$ \\
\hline
\end{tabular}

The top plot of Fig. 3 shows the millisecond-level Type 1 clock jumps, corresponding to the $26 \mathrm{~ms}$ accumulated code clock jump in Table 3. The magnitude of the phase clock jump was zero in the entire duration. The bottom plot of Fig. 3 shows the microsecond-level Type 2 clock jumps, corresponding to the $48 \mu$ s accumulated phase clock jump in Table 3 . The magnitude of the code clock jump was zero in the entire duration. Table 3 shows that $100 \%$ detection and correction of all clock jump types was achieved by the proposed algorithm. The algorithm did not produce any false detection in these tests. 

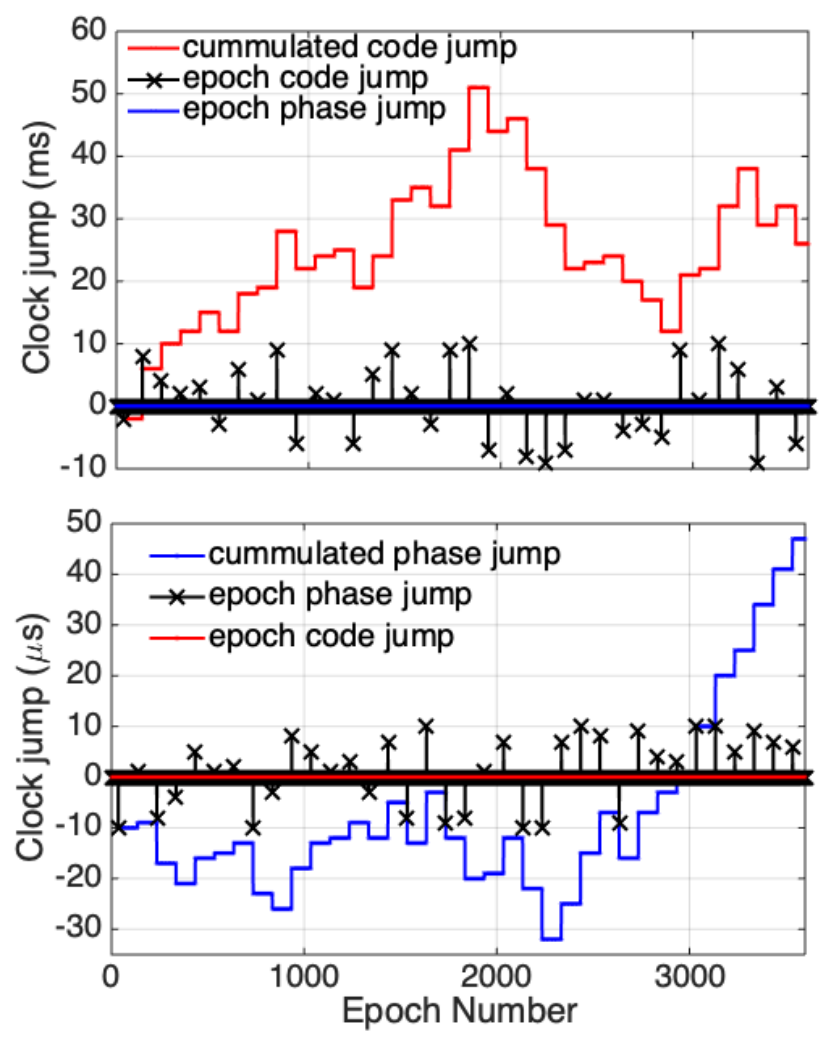

Fig. 3 Plots of simulated epochs' clock jumps and the cumulated clock jump at different epochs: Type 1 millisecond-level clock jump (top); and Type 2 microsecond-level clock jump (bottom).

The performance of the algorithm in handling the simultaneous occurrence of clock jumps and cycle slips on satellites' phase observations was also investigated. For this test, the previous cycle slip simulation for the uBlox single-frequency receiver was repeated completely, i.e. having the same cycle slip values at the same epochs, while a new set of clock jump values were simulated starting from the $30^{\text {th }}$ epoch and at 100 -epoch intervals. In this way, there were 36 epochs out of the 3600 epochs of observations, where, simultaneously, clock jumps and cycle slips occurred on at least 1 or up to $\mathrm{N}$ observed satellites. Table 4 shows that for a Type 1 clock jump of millisecond-level, the detection and correction of clock jumps was $100 \%$, while a $100 \%$ detection and $99.8 \%$ correct fixing of cycle slips, as previously obtained, were recorded. These indicate the independence of code clock jumps from phase cycle slips. The Table 4 also shows that the same results were obtained when the same millisecond-level but Type 2 clock jump occurred simultaneously with cycle slips. 
Table 4 Results of simulated cycle slips and clock jumps on the u-blox single-frequency receiver

\begin{tabular}{|c|c|c|c|c|c|c|c|}
\hline $\begin{array}{c}\text { Clock } \\
\text { jump } \\
\text { type }\end{array}$ & $\begin{array}{c}\sum \\
\text { jump }\end{array}$ & $\begin{array}{c}\text { Jump } \\
\text { detection } \\
\text { and } \\
\text { correction }\end{array}$ & $\begin{array}{l}\text { Clock } \\
\text { jump } \\
\text { range }\end{array}$ & $\begin{array}{l}\text { Sim. } \\
\text { Cycle } \\
\text { slip } \\
\text { range }\end{array}$ & $\begin{array}{c}\text { Quantity } \\
\text { determined. }\end{array}$ & $\begin{array}{l}\text { Quantity } \\
\text { fixed }\end{array}$ & $\begin{array}{c}\text { Max. } \\
\text { fix } \\
\text { error } \\
\text { (cycles) }\end{array}$ \\
\hline $\begin{array}{c}\text { Type } \\
1\end{array}$ & $42 \mathrm{~ms}$ & $\begin{array}{c}\text { Det: }(100 \%) \\
\text { Cor: }(100 \%) \\
\text { False }=0\end{array}$ & $\begin{array}{l}-10 \text { to } \\
10 \mathrm{~ms}\end{array}$ & $\begin{array}{c}-100 \\
\text { to } \\
+100 \\
\text { cycles }\end{array}$ & $\begin{array}{c}4480 \\
(100 \%)\end{array}$ & $\begin{array}{c}4469 \\
(99.8 \%)\end{array}$ & +1 \\
\hline $\begin{array}{c}\text { Type } \\
2\end{array}$ & $42 \mathrm{~ms}$ & $\begin{array}{c}\text { Det: }(100 \%) \\
\text { Cor: }(100 \%) \\
\text { False }=0\end{array}$ & $\begin{array}{l}-10 \text { to } \\
10 \mathrm{~ms}\end{array}$ & $\begin{array}{c}-100 \\
\text { to } \\
+100 \\
\text { cycles }\end{array}$ & $\begin{array}{c}4480 \\
(100 \%)\end{array}$ & $\begin{array}{c}4469 \\
(99.8 \%)\end{array}$ & +1 \\
\hline $\begin{array}{c}\text { Type } \\
3\end{array}$ & $32 \mu \mathrm{s}$ & $\begin{array}{c}\text { Det: }(100 \%) \\
\text { Cor: }(100 \%) \\
\text { False }=0\end{array}$ & $\begin{array}{l}-10 \text { to } \\
10 \mu \mathrm{s}\end{array}$ & $\begin{array}{c}-100 \\
\text { to } \\
+100 \\
\text { cycles }\end{array}$ & $\begin{array}{c}4480 \\
(100 \%)\end{array}$ & $\begin{array}{c}4469 \\
(99.8 \%)\end{array}$ & +1 \\
\hline $\begin{array}{c}\text { Type } \\
2\end{array}$ & $8 \mu \mathrm{s}$ & $\begin{array}{c}\text { Det: }(100 \%) \\
\text { Cor: }(100 \%) \\
\text { False }=1\end{array}$ & $\begin{array}{l}-10 \text { to } \\
10 \mu \mathrm{s}\end{array}$ & $\begin{array}{c}-1600 \\
\text { to } \\
+1600 \\
\text { cycles }\end{array}$ & $\begin{array}{c}4482 \\
(100 \%)\end{array}$ & $\begin{array}{c}4451 \\
(99.3 \%)\end{array}$ & +1575 \\
\hline
\end{tabular}

For the microsecond-level Type 3 clock jumps in the presence of cycle slips, the algorithm achieved the same detection and correction results. In these tests, the cycle slip values were within the range of -100 to 100 cycles.

In the final test, we simulated cycle slip values between -1600 to 1600 cycles to create cycle slips of similar magnitude, in distance units, to $1 \mu$ s clock jumps ( $1 \mu \mathrm{s} \approx 1575$ cycles). 
The result is shown in the last row of Table 4. We observed that the algorithm achieved $100 \%$ detection and correction of the microsecond-level clock jumps, but with 1 false clock jump detection at one of the 115 epochs (see Fig. 4) when all satellites had cycle slips. The 10 cycle slip values of the 10 slipped satellites at the false-detection epoch were large numbers around the equivalent of $1 \mu \mathrm{s}$, and the algorithm interpreted it as a clock jump of $1 \mu \mathrm{s}$, thus leading to 10 common fixed error of +1575 cycles. Also, in 2 of these 115 epochs, the 11 detected cycle slips in each of the epochs were wrongly fixed by a fixing error of +1 cycle. These three occurrences resulted in the achieved $99.3 \%$ of correct fixes. However, the algorithm was able to detect and fix all cycle slips and clock jumps correctly in the 7 epochs when all observed satellites had cycle slips simultaneously with clock jumps.

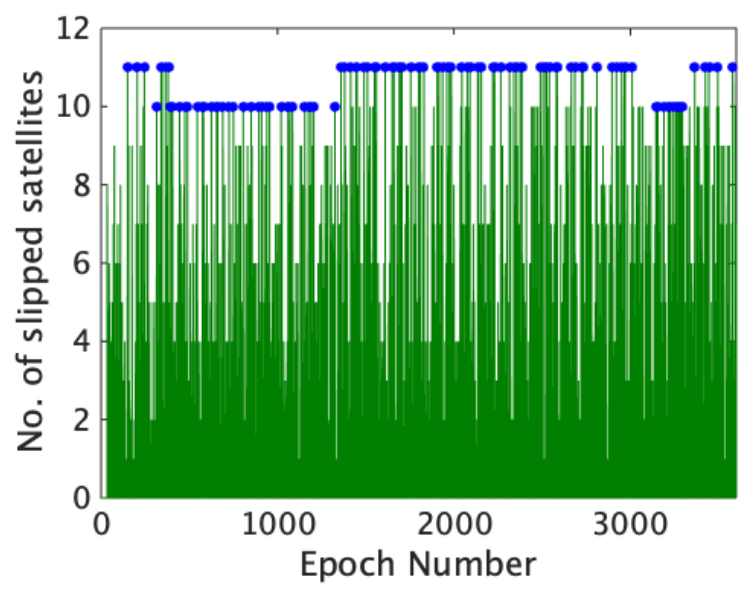

Fig. 4 Plots of number of cycle-slipped satellites at all epochs when cycle slips were simulated in the presence of microsecond-level Type 2 clock jumps. The blue circles indicate the 115 epochs that all satellites observed had cycle slips, out of which 113 were correctly fixed.

The results indicate that when there is a wrong fix, which mostly happens when all satellites' phase observations have cycle slips, the proposed algorithm may produce a small, common fix error, usually between \pm 2 cycles on all determined cycle slip values as $\varepsilon_{c k}$ may not have been accurately obtained and eliminated. Moreover, false detection of a clock jump of magnitude, say $1 \mu \mathrm{s}$, may only happen for a Type 2 (not for Type 1 or 3 ) clock jump when all the satellites' have cycle slips in the range of \pm 1600 cycles. A false clock detection would degrade positioning as much as a missed detection. Undetected or falsely detected millisecond-level jumps can cause relatively large positioning errors, but frequent microsecond level jumps, if not detected, would also cause smaller, but more frequent 
positioning errors. Cycle slips, depending on their magnitude, may interfere with both millisecond and microsecond-level clock jump detection.

\section{Conclusion}

We propose an algorithm capable of detecting and correcting cycle slips on phase observations and all types of receiver clock jumps on code and phase observations in realtime. The algorithm, which is designed to be implemented on any one band of a GNSS receiver, is essentially targeted at facilitating precise point positioning and timing with a single-frequency receiver thus driving a cheaper alternative to the current use of expensive dual-frequency receivers for the same purpose. The algorithm is simple; it is based on successive differencing, statistical extraction and thresholding, estimation by weighted least square adjustment and simple rounding-up of float values. The performance of the algorithm was investigated with different ranges of cycle slip values and for different types of receiver clock jumps, using data acquired by different GNSS receivers driven by both quartz crystal and atomic frequency standard clocks. The results indicated that the algorithm achieves $100 \%$ detection and correction of all types of clock jumps at both millisecond- and microsecondlevel; between 97 to $100 \%$ cycle slip detection; and between 96.9 to $100 \%$ cycle slip correction, when simulated CJCS were applied on different rates observation acquired by different fixed and mobile GNSS receivers. The algorithm shows good performance for both high and low rate data sets using data sets obtained by different receivers driven by an atomic frequency standard and quartz crystal oscillators, but achieved better performance with high rate data than with low rate data. The results indicate a potential for improving precise point positioning cheaply with a single-frequency receiver and not just with an expensive multi-frequency one - a driver for low-cost quality positioning with phase measurements in cheap single-frequency GNSS devices. Future work would use real-life cycle-slip affected data sets obtained by receivers having both millisecond- and microsecondlevel clock jumps.

\section{References}

Cosser E, Hill J C, Roberts W G, Meng X, Moore T, Dodson H A (2004) Bridge monitoring with Garmin handheld receivers. In 1st FIG International Symposium on 
Engineering Surveys for Construction Works and Structural Engineering, Nottingham, United Kingdom, June 28 - July 1

de Lacey M C, Reguzzoni M, Sanso F (2011) Real-time cycle slip detection in triple frequency GNSS. GPS Solutions 16(3):353-362. https://doi.org/10.1007/s10291-011$0237-5$

Deo M, El-Mowafy A (2015) Cycle slip and clock jump repair with multifrequency multi-constellation GNSS data for precise point positioning. In: Proc International Global Navigation Satellite Systems Society (IGNSS) Symposium 2015, Outrigger Gold Coast, Australia, July 14-16, pp 1-15

Fath-Allah F. T (2010) A new approach for cycle slips repairing using GPS single frequency data. World Applied Sciences Journal 3(8):315-325

Guo F. Zhang X(2014) Real-time clock jump compensation for precise point positioning. GPS Solutions 18(1):41-50. https://doi.org/10.1007/s10291-012-0307-3

Jia P, Wu L(2001) An algorithm for detecting and estimating cycle slips in single-frequency GPS. Chinese Astronomy and Astrophysics 2001(25):515-521. https://doi.org/10.1016/S0275-1062(01)00105-9

Kim D, Langley B R(2002) Instantaneous real-time cycle-slip correction for quality control of GPS carrier-phase measurements. Navigation 49(4):205-222. https://doi.org/10.1002/j.2161-4296.2002.tb00269.x

Markovsky I, Huffel S V (2007) Overview of total least-squares methods, Signal Processing 87(10):2283-2302. https://doi.org/10.1016/j.sigpro.2007.04.004

Momoh J A (2013) Robust GNSS point positioning in the presence of cycle slips and observation gaps. Ph.D. Dissertation, University College London, United Kingdom

Momoh J A, Ziebart M (2012) Instantaneous cycle slip detection, code multipath mitigation and improved ionospheric correction for enhanced GPS single-frequency. In: Proc. ION GNSS 2012, Institute of Navigation, Nashville, Tennessee, USA, September 17-21, pp 1217 - 1232

Ren Z, Li L, Zhong J, Zhao M, Shen Y (2011). A real-time cycle-slip detection and repair method for single frequency GPS receiver. In 2nd International Conference on Networking and Information Technology IPCSIT 2011, Hong Kong, pp 224-230 
Weinbach U. and Schon S. (2011) GNSS receiver clock modeling when using high-precision oscillators and its impact on PPP. Advances in Space Research 47(2):229-238. https://doi.org/10.1016/j.asr.2010.06.031

\section{Authors Biographies}

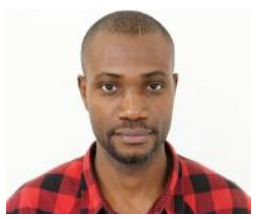

John A. Momoh is a space systems engineer with the National Space Research and Development Agency, Nigeria, and a visiting lecturer, Department of Electronic and Information Engineering, Landmark University, Nigeria. He obtained BSc and MSc degrees in electronic and electrical engineering in 1998 and 2005 respectively, from the Obafemi Awolowo University, Nigeria. He also obtained a Master degree in Navigation and Related Applications in 2006 from the Politecnico di Torino, Italy, and a Ph.D. degree in Satellite Navigation, University College London, UK, in 2013. His current research focuses on signal processing and algorithm developments for mitigating GNSS errors and improving positioning.

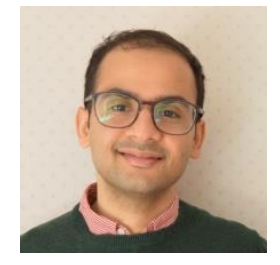

Santosh Bhattarai is a lecturer in space geodesy in the Department of Civil, Environmental and Geomatic Engineering (CEGE) at University College London (UCL) with research interests in astrodynamics and space debris dynamics for orbit prediction and orbit determination, and also, in various aspects of global navigation satellite systems (GNSS) and related technologies. He was awarded a Ph.D. in Global Navigation Satellite Systems from UCL in 2015 and a BSc in Mathematics and Physics from the University of York in 2009.

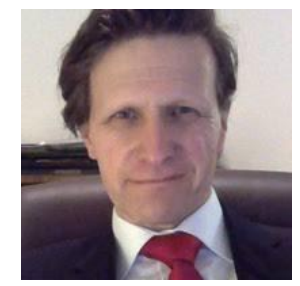

Marek Ziebart is a professor of space geodesy and the Director of the Space Geodesy and Navigation Laboratory (SGNL) at the University College London, United Kingdom. He specializes in orbit prediction and orbit determination of various classes of space vehicles, and in various aspects of global navigation satellite systems. 
